\title{
A crucial role for plasmacytoid dendritic cells in antiviral protection by CpG ODN-based vaginal microbicide
}

\author{
Hong Shen and Akiko Iwasaki
}

Section of Immunobiology, Yale University School of Medicine, New Haven, Connecticut, USA.

\begin{abstract}
Topical microbicides represent a promising new approach to preventing HIV and other sexually transmitted infections. TLR agonists are ideal candidates for microbicides, as they trigger a multitude of antiviral genes effective against a broad range of viruses. Although vaginal application of $\mathrm{CPG}$ oligodeoxynucleotides (ODNs) and poly I:C has been shown to protect mice from genital herpes infection, the mechanism by which these agents provide protection remains unclear. Here, we show that plasmacytoid DCs (pDCs) are required for CpG ODNmediated protection against lethal vaginal challenge with herpes simplex virus type 2 (HSV-2). Moreover, we demonstrate that cells of both the hematopoietic and stromal compartments must respond to CpG ODN via TLR9 and to type I IFNs through IFN- $\alpha \beta$ receptor (IFN- $\alpha \beta$ R) for protection. Thus, crosstalk between pDCs and vaginal stromal cells provides for optimal microbicide efficacy. Our results imply that temporally and spatially controlled targeting of $\mathrm{CPG}$ ODN to $\mathrm{pDCs}$ and epithelial cells can potentially maximize their effectiveness as microbicides while minimizing the associated inflammatory responses.
\end{abstract}

\section{Introduction}

Sexually transmitted infections (STIs) such as HIV-1 and herpes simplex virus type 2 (HSV-2) represent a major public health concern. Currently, there are no effective vaccines against STI causative agents. In the absence of effective vaccines, the development of a safe and effective topical microbicide to prevent the sexual transmission of STI could play a major role in world-wide reduction of STI transmission and potentially save millions of lives lost to HIV-1 (1). A promising topical microbicidal approach is the use of agents to trigger a nonspecific broad antiviral state in the genital mucosa prior to encounter with sexually transmittable viruses. In this regard, the TLR agonists represent an ideal candidate that can induce an antiviral state. The innate immune system has evolved to recognize pathogens that enter through a variety of mucosal surfaces and to induce a rapid cascade of events leading to antimicrobial defense (2). Various TLRs are expressed by a variety of cell types present in the genital mucosa $(3,4)$. Agonists of TLR9 (CpG oligodeoxynucleotides [ODNs]) (5-7) and TLR3 (poly I:C) (4) have been used successfully to provide complete protection against HSV-2 in the mouse model of genital herpes.

Intravaginal (ivag) inoculation of $\mathrm{CPG}$ ODN has been shown to induce several changes in the vaginal mucosa. First, CPG ODN induces active proliferation of the genital epithelium (5). Second, a variety of inflammatory cells have been shown to accumulate in vaginal mucosa, including $\mathrm{NK}$ cells, $\mathrm{CD} 11 \mathrm{~b}^{+} \mathrm{F} 4 / 80^{+}$myeloid cells, and CD $11 \mathrm{~b}^{+} \mathrm{CD} 11 \mathrm{c}^{+} \mathrm{DCs}(5,8)$. There are conflicting observations regarding the roles of T cells and IFN- $\gamma$. A previous study has shown that the protection requires IFN- $\gamma$, IL-12, IL-18, and $\mathrm{T}$ cells (6) while others have demonstrated that complete protection is conferred by CPG ODN in the absence of any lymphocytes

Nonstandard abbreviations used: HSV-2, herpes simplex virus type 2; IFN- $\alpha \beta R$ IFN- $\alpha \beta$ receptor; ivag, intravaginal(ly); ODN, oligodeoxynucleotide; $\mathrm{pDC}$,

plasmacytoid DC; STI, sexually transmitted infection.

Conflict of interest: The authors have declared that no conflict of interest exists. Citation for this article: J. Clin. Invest. 116:2237-2243 (2006). doi:10.1172/JCI28681.
$\left(\mathrm{RAG}^{-/-} \gamma \mathrm{c}^{-/-}\right)$(9) or IFN- $\gamma$ (5). However, the precise cellular and molecular mechanism by which $\mathrm{CPG}$ ODN provides protection against HSV-2 challenge is unclear, which may hinder the clinical application of CPG ODN as a microbicide.

In this study, we aim to address several key questions with respect to the mechanism of $\mathrm{CPG}$ ODN-mediated antiviral protection. First, what are the critical cell types that recognize $\mathrm{CpG}$ ODN and mediate its ability to induce an antiviral state, keratinocytes or hematopoietic cells? The first cells to come in contact with $\mathrm{CPG} O \mathrm{ODN}$ are the keratinocytes lining the vaginal cavity. The importance of direct recognition of $\mathrm{CPG}$ ODN by the vaginal keratinocytes in vivo is unknown. In addition, it is not clear whether recognition of $\mathrm{CPG}$ ODN by hematopoietic cells is required for protection. Second, what is the significance of the recruitment of various DC types to the vaginal mucosa in providing protection against HSV-2? Third, are type I IFNs, the most potent set of antiviral cytokines, required for $\mathrm{CPG}$ ODN-mediated protection against HSV-2? Fourth, if type I IFNs are involved in generating an antiviral state in the host, do they act on the keratinocytes, which are the primary target of HSV-2 infection (10), or do they act on hematopoietic cells and induce their antiviral activity?

To address these issues, using irradiation-induced BM chimeric mice, we examined the importance of various cell types and their responsiveness to $\mathrm{CPG}$ ODN in conferring an antiviral state in the host. Further, we demonstrate that type I IFNs, but not type II IFN or IL-12, play a critical role in CPG ODNmediated protection and that both stromal and hematopoietic compartments must respond to type I IFNs for this process. We show that depletion of individual lymphocyte subsets (CD4, CD8, or NK cells) prior to CPG ODN treatment does not compromise protection. Finally, we demonstrate that plasmacytoid DCs (pDCs) are recruited to the site of CPG ODN inoculation and that they play a critical role in $\mathrm{CPG} O \mathrm{ODN}$-mediated protection. These results imply that pDCs might be exploited to confer a general state of antiviral innate immunity against a variety of STIs within the genital mucosa. 

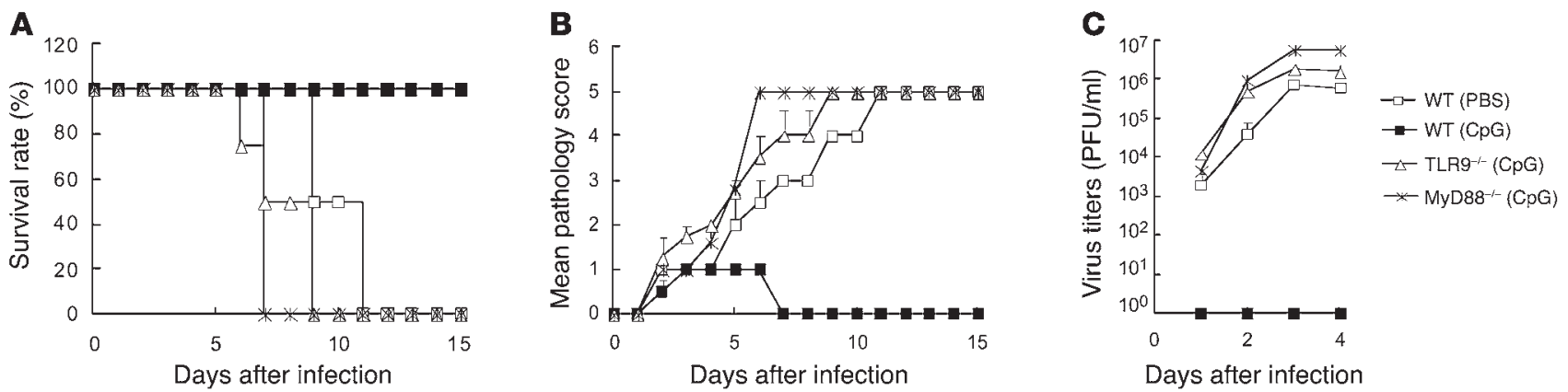

Figure 1

Requirement of TLR9 and MyD88 for CpG ODN-mediated protection from genital HSV-2 challenge. (A) Survival rates, (B) genital mean pathology scores, and (C) virus titers in vaginal washes of mice inoculated ivag with PBS or CpG ODN 24 hours prior to challenge with $10^{4}$ PFU of HSV-2. The above parameters were examined daily after infection. Values represent mean \pm SEM $(n=5 / g r o u p)$. Data are representative of 2 repeated experiments.

\section{Results}

TLR9 and MyD88 are required for antiviral protection conferred by vaginal CPG ODN. To examine the importance of TLR9 signaling in antiviral protection by $\mathrm{CpG}$ ODN, mice deficient for either TLR9 or MyD88 were inoculated ivag with CPG ODN and, 24 hours later, challenged with a lethal dose $\left(10^{4} \mathrm{PFU}\right)$ of HSV-2. As expected, both TLR9 and the obligate adapter MyD88 (11) were required to confer protection upon $\mathrm{CPG}$ ODN inoculation (Figure 1). Thus, TLR9 $^{-/-}$and $\mathrm{MyD} 88^{-/-}$mice failed to control local viral replication (Figure 1C), consequently developing rapid pathology (Figure 1B) and succumbing to death (Figure 1A). As reported previously (5-7), WT mice were completely protected from challenge by pretreatment with CPG ODN. These data provided us with the basis from which to examine the importance of different cell types in conferring CpG ODN-induced innate protection against HSV-2.

Antiviral protection requires both hematopoietic and stromal cell responses to $C p G O D N$. It is known that cells found within the vaginal epithelial layer express TLR9 $(3,4)$. In addition, in purified vaginal keratinocytes, TLR9 was expressed at a low level, which was upregulated upon CpG ODN stimulation (Supplemental Figure 1; supplemental material available online with this article; doi:10.1172/JCI28681DS1). To elucidate the cell types involved in the $\mathrm{CpG} O \mathrm{ODN}$-mediated innate protection against HSV-2, 2 sets of irradiation-induced BM chimeric mice were generated. To examine the importance of CPG ODN recognition by the hematopoietic cells in providing protection, the BM from TLR9-/- mice was used to reconstitute irradiated WT mice (TLR9 $-/-\rightarrow$ WT). Upon vaginal delivery of CPG ODN, these mice succumbed to viral infection and death, with similar kinetics seen in the TLR9 $/-$ mice (Figure 2). These results indicate that hematopoietic cells must respond through their TLR9 in order to provide antiviral protection against HSV-2 following CPG ODN microbicidal treatment. Next, the role of $\mathrm{CPG}$ ODN recognition by the radioresistant stromal cells was examined by generating WT $\rightarrow$ TLR9-/- chimera. Interestingly, these mice also succumbed to HSV-2-induced disease, albeit with delayed kinetics compared with the TLR9-/ $\rightarrow$ WT chimeric mice (Figure 2), indicating that stromal cell responsiveness to TLR9 is important in CPG ODN microbicidal activity. This delay correlated with reduced viral titers found at days 2 and 3 after infection in the vaginal washes of WT $\rightarrow$ TLR9-/- chimera compared with those of TLR9 $-/ \rightarrow$ WT chimeric mice $(P<0.05$, Figure $2 \mathrm{C})$. Thus, these results indicate that both hematopoietic and stromal compartments must respond to $\mathrm{CPG} O \mathrm{ODN}$ in order to confer antiviral protection against HSV-2 challenge and that the effect was more significant when hematopoietic cells' responsiveness to CPG ODN was ablated compared with that of the stromal cells.

$p D C$ s are required for $C p G O D N$ protection against $H S V$-2. It is known that a variety of cell types of the lymphoid and myeloid lineages are recruited to the vaginal mucosa following $\mathrm{CPG}$ ODN inoculation
A

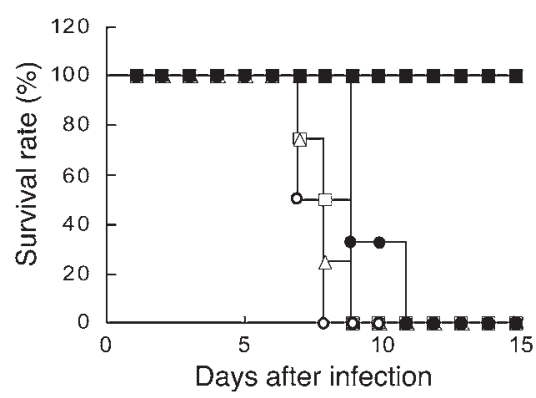

B

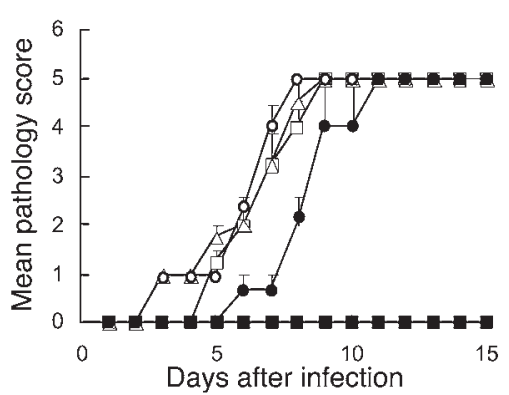

C

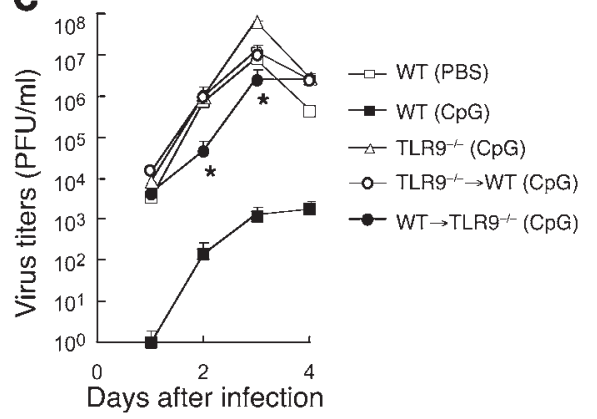

Figure 2

Recognition of CpG ODN via TLR9 by both the hematopoietic and stromal cells is required for protection from genital HSV-2 challenge. WT ( $n=5$ ), TLR9 $^{--} \rightarrow$ WT $(n=6)$, and WT $\rightarrow$ TLR9 $^{-1-}(n=3)$ chimeric mice were inoculated ivag with PBS or CpG ODN 24 hours prior to challenge with $10^{4}$ PFU of HSV-2. (A) Survival rates, (B) genital mean pathology scores, and (C) virus titers in vaginal washes of mice were monitored. Values represent

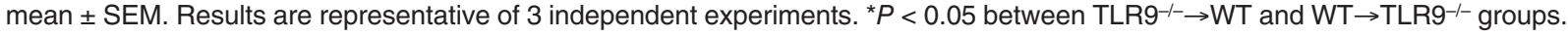



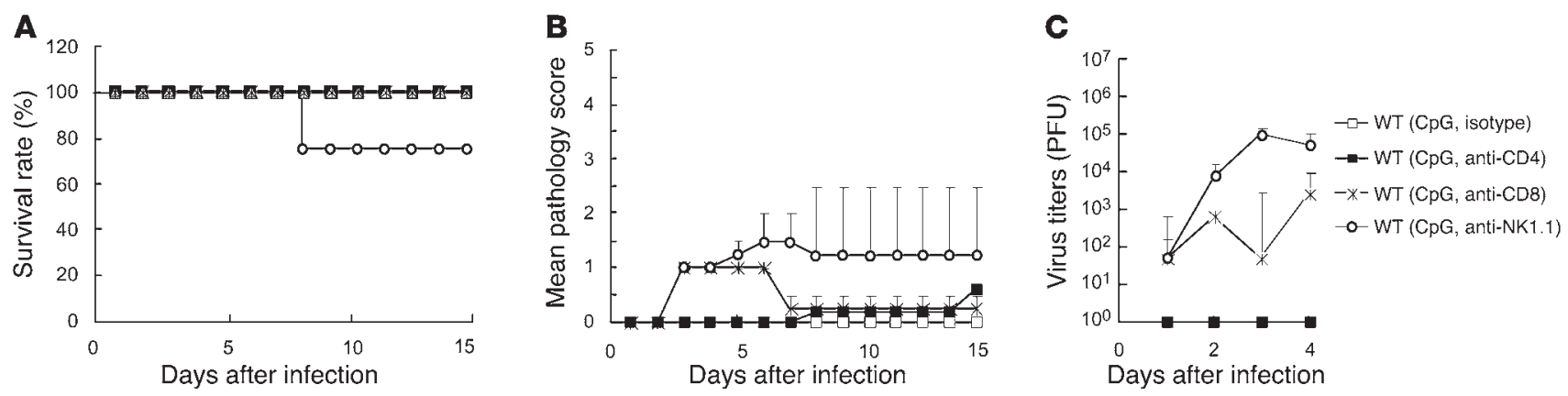

Figure 3

Lymphocyte populations are not required for CpG ODN-mediated protection against genital HSV-2 challenge. Mice were treated with antibodies to deplete CD4+ T cells, CD8 ${ }^{+}$T cells, or NK cells prior to CpG ODN inoculation and during the course of the experiment. (A) Survival rates, (B) genital mean pathology scores, and (C) virus titers in vaginal washes of mice were monitored following CpG ODN ivag inoculation and HSV-2 challenge ( $\left.10^{4} \mathrm{PFU}\right)$. Values represent mean $\pm \operatorname{SEM}(n=5 /$ group $)$. Data are representative of 3 independent experiments.

(5). To identify the hematopoietic cell type responsible for CpG ODN-mediated protection, different subsets of cells of the innate and adaptive immune system were depleted prior to and following $\mathrm{CpG}$ ODN treatment and challenge with HSV-2. These results revealed that $\mathrm{CD} 4^{+} \mathrm{T}$ cells or $\mathrm{CD}^{+} \mathrm{T}$ cells contribute minimally to protection against genital herpes (Figure 3 ). While there was a modest increase in the viral titers and pathology in mice depleted of NK cells following CpG ODN microbicide delivery, the majority of NK-depleted mice were still protected from viral challenge (Figure 3A). All of these depletion protocols resulted in more than $90 \%$ depletion of the targeted cell subset (Supplemental Figure 2).

Having ruled out the role of these lymphocytes in critically providing $\mathrm{CPG}$ ODN-mediated innate protection, we focused on the role of pDCs. The pDCs express TLR9 and respond to CpG ODN and are potent secretors of the antiviral cytokines type I IFNs (12, 13). First, we examined the presence of pDCs in the vaginal mucosa prior to and following CpG ODN treatment. pDCs were found in small numbers in the vaginal mucosa at steady state and were recruited rapidly following ivag CPG ODN delivery (Figure 4). Next, to examine the importance of pDCs in the context of CPG ODN-based microbicides, mice were depleted of $\mathrm{pDCs}$ by Abs prior to and following inoculation of $\mathrm{CPG} O \mathrm{ODN}$, and these mice were challenged with a lethal dose of HSV-2. Mice depleted of pDCs were remarkably susceptible to viral infection and disease (Figure 5). These mice harbored high viral titers in the vagina, even more so than the WT mice not treated with CPG ODN (Figure 5C), suggesting that pDCs present in WT mice are able to limit viral replication even in the absence of $\mathrm{CPG}$ ODN priming. Further, pDC-depleted mice suffered from disease almost comparable to that of non-CpG ODN-treated WT mice (Figure 5B). As a consequence, most of the pDC-depleted mice succumbed to death (Figure 5A) albeit with delayed kinetics compared with the TLR9- $/-\rightarrow$ WT chimeric mice (Figure 2A). The fact that some pDC-depleted mice survived may reflect incomplete pDC depletion (>90\% depletion; Supplemental Figure 2) or indicate that a hematopoietic, non-pDC cell type contributes to the residual protection against viral infection.

The importance of type IIFNs in CPG ODN-induced antiviral state. pDCs represent the most potent secretors of type I IFNs in response to a variety of viral and synthetic stimuli, including CPG ODN $(12,13)$. The type I IFNs are the best characterized antiviral cytokines capable of inhibiting replication of most viruses $(14,15)$. Our data on the requirement for $\mathrm{pDCs}$ in protection offered by the $\mathrm{CPG}$ ODN-based microbicide suggest the importance of type I IFNs. To examine this possibility, mice deficient in IFN- $\alpha \beta$ receptor (IFN- $\alpha \beta R$ ) were inoculated vaginally with $\mathrm{CPG}$ ODN and, 24 hours later, challenged with the lethal dose of HSV-2. This analysis showed that CpG ODN completely failed to provide protection from HSV-2 challenge in IFN $\alpha \beta \mathrm{R}^{-/-}$mice (Figure 6). Thus, IFN- $\alpha \beta$ is required to confer protection following vaginal $\mathrm{CPG}$ ODN treatment. In contrast, mice deficient in other key antiviral cytokines, such as IL-12 and IL-23

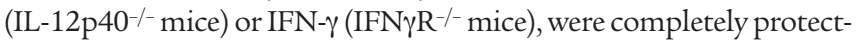
ed from HSV-2 challenge following CpG ODN treatment (Figures 7 and 8). Collectively, our data thus far suggest that $\mathrm{pDCs}$ are required to secrete type I IFNs at the site of infection and prevent replication of the virus in the vaginal epithelial cells via IFN- $\alpha \beta R$, which is the main target of primary HSV-2 infection and replication (16).

Both stromal and hematopoietic cell responsiveness to type I IFNs are required for protection. To elucidate the importance of IFN responsiveness by the vaginal keratinocytes, we generated irradiation-induced

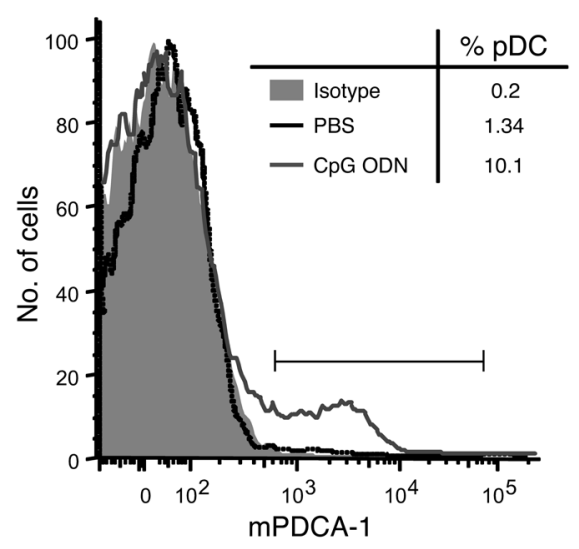

\section{Figure 4}

pDCs are recruited to vaginal mucosa 24 hours after CpG ODN treatment. Single-cell suspensions of the vaginal tissue were prepared and stained with anti-mPDCA-1 Ab, and live cells were gated by exclusion of 7-AAD and analyzed by flow cytometry. Shaded area indicates isotype control $\mathrm{Ab}$ labeling of cells isolated from CpG ODN-treated mice; black line indicates vaginal cells from mice treated with PBS and stained with anti-mPDCA-1; and gray line indicates vaginal cells from mice treated with CpG ODN 24 hours earlier. Data are representative of 4 independent experiments. 

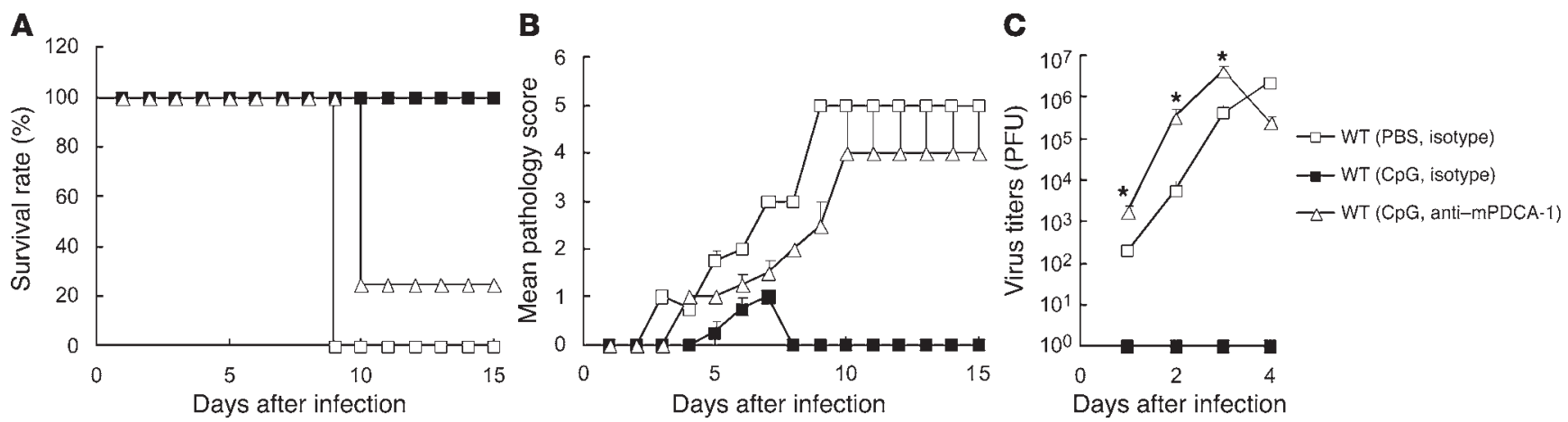

Figure 5

pDCs are critical in CpG ODN-mediated protection from genital HSV-2 challenge. WT mice were treated with anti-mPDCA-1 or isotype control on -1 and 1 day of CpG ODN treatment and were challenged ivag with 104 PFU of HSV-2 24 hours following CpG ODN inoculation. (A) Survival rates, (B) genital mean pathology scores, and (C) virus titers in vaginal washes of mice were monitored. Values represent mean \pm SEM ( $n=5$ /group). Results are representative of 2 separate experiments. ${ }^{*} P<0.05$ between PBS-treated (open squares) and pDC-depleted, CpG ODN-treated (open triangles) groups.

chimeric mice that lack IFN- $\alpha \beta$ R on the radioresistant stromal cells but retain intact IFN- $\alpha \beta$ R expression on the hematopoietic cells $\left(\mathrm{WT} \rightarrow \mathrm{IFN} \alpha \beta \mathrm{R}^{-/-}\right)$. Upon vaginal treatment with $\mathrm{CpG}$ ODN, these mice were challenged with HSV-2, and disease progression was monitored. To our surprise, the lack of IFN- $\alpha \beta R$ on stromal cells, including vaginal epithelial cells, did not severely compromise these mice from CPG ODN-mediated protection against HSV-2. Thus, improved survival (Figure 6A) and pathology (Figure 6B) were observed in the $\mathrm{CPG}$ ODN-treated WT $\rightarrow \mathrm{IFN} \alpha \beta \mathrm{R}^{-/-}$mice compared with the IFN $\alpha \beta \mathrm{R}^{-/-}$mice. Further, control of local viral replication remained intact when the hematopoietic cells, including pDCs, expressed IFN- $\alpha \beta R$ (Figure $6 \mathrm{C}$ ). These results led us to examine the importance of IFN responsiveness in the hematopoietic cells in CpG ODN-mediated protection. To address this issue, BM cells of the IFN $\alpha \beta \mathrm{R}^{-/-}$mice were used to reconstitute lethally irradiated WT mice $\left(\mathrm{IFN} \alpha \beta \mathrm{R}^{-/-} \rightarrow \mathrm{WT}\right)$. Upon reconstitution, these chimeric mice were treated with CPG ODN and challenged with HSV-2. IFN responsiveness by the hematopoietic cells was also found to be required for $\mathrm{CPG}$ ODN-mediated protection against HSV-2 (Figure 6). These data indicate that stromal cell responsiveness to type I IFNs was not sufficient and that BM-derived cells must also respond to IFN- $\alpha \beta$ in order for $\mathrm{CPG}$ ODN-based microbicide to provide antivi- ral protection. In fact, IFN responsiveness by the hematopoietic cells appeared to be slightly more important than that of the stromal cells in providing control of viral replication (Figure 6C) and subsequent protection from disease (Figure $6, \mathrm{~A}$ and $\mathrm{B}$ ).

\section{Discussion}

The innate immune system is designed to detect a variety of pathogens through multiple pattern recognition receptors and to induce optimal sets of antiviral or antibacterial genes to provide immediate defense against invading pathogens $(17,18)$. By engaging the appropriate TLRs, a broad antiviral state can be achieved in the host. In this study, we dissected the mechanisms by which the ivag inoculation of $\mathrm{CPG}$ ODN provides protection against subsequent challenge by HSV-2. We showed that CPG ODN responsiveness via TLR9 is required by both the hematopoietic and stromal compartments for protection. We demonstrated that $\mathrm{pDCs}$, but not NK cells, CD4 T cells, or CD8 T cells, are required for CpG ODN to protect the host from HSV-2. Further, type I IFNs were required for protection. The type I IFNs induced as a result of vaginal CPG ODN inoculation must act on both the stromal and hematopoietic compartments to suppress viral replication and prevent disease. The mice lacking IFN- $\alpha \beta$ R from the hematopoietic cells had
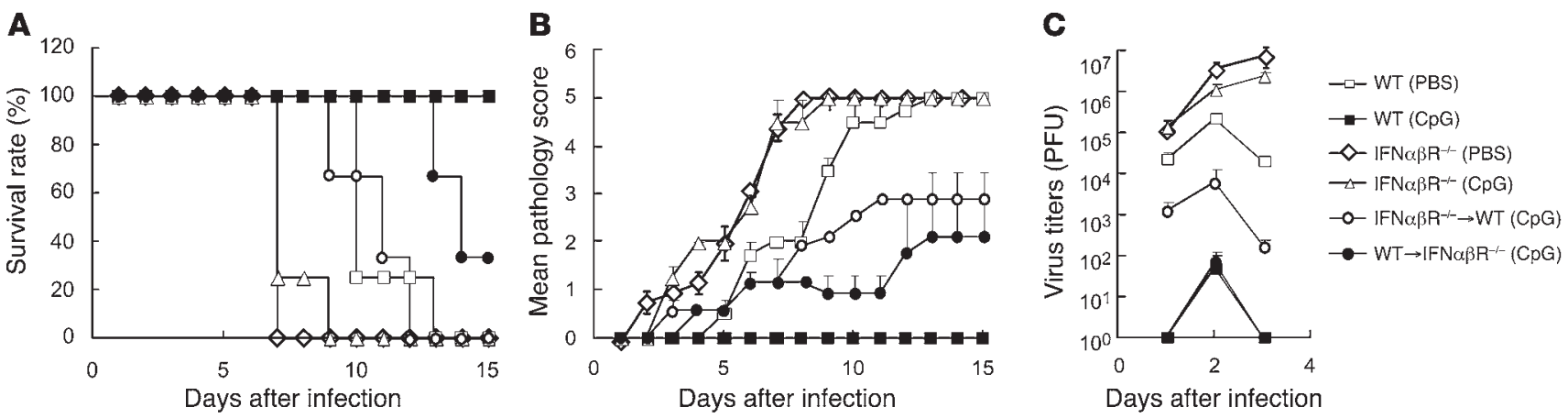

Figure 6

Responsiveness to type I IFNs is required in both the stromal and hematopoietic cells for complete protection from lethal challenge of genital HSV-2 following CpG ODN treatment. (A) Survival rates, (B) genital mean pathology scores, and (C) virus titers in vaginal washes were monitored in IFN $\alpha \beta R^{-1-}$ mice, WT (129 background) mice, and IFN $\alpha \beta R^{-1-} \rightarrow$ WT or WT $\rightarrow I F N \alpha \beta R^{-/-}$chimeric mice following CpG ODN ivag inoculation and HSV-2 challenge (104 PFU). Values represent mean $\pm \operatorname{SEM}(n=4 /$ group$)$. 

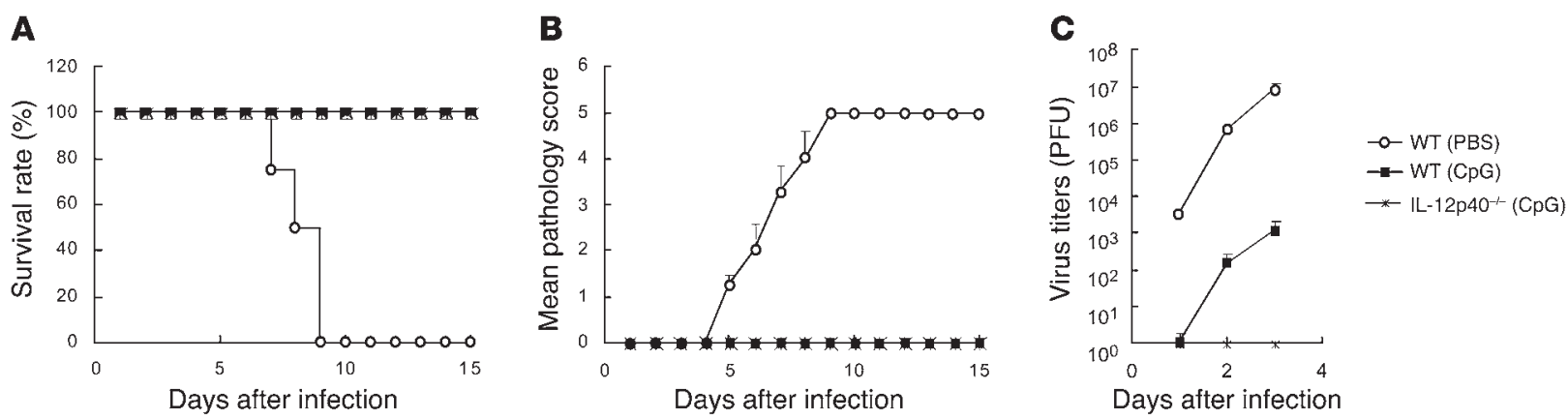

Figure 7

IL-12 and IL-23 are not required for CpG ODN-mediated protection against genital HSV-2 challenge. (A) Survival rates, (B) genital mean pathology scores, and (C) virus titers in vaginal washes of WT (C57BL/6) and IL-12p40-/- (C57BL/6 background) mice were monitored following CpG ODN ivag inoculation and HSV-2 challenge (104 PFU). Values represent mean \pm SEM $(n=5 /$ group). Data are representative of 2 separate experiments.

a more severe phenotype than those lacking IFN- $\alpha \beta R$ from the stromal cells, including the vaginal keratinocytes.

We showed that $\mathrm{CPG} O \mathrm{ODN}$-mediated protection did not require T or NK cells, IFN- $\gamma$ R, or IL-12p40. These data differ from the findings of a previous study that demonstrated that SCID and nude mice succumbed to HSV-2 challenge despite CpG ODN treatment (6). Although we do not know the reasons for this discordance, it may relate to the use of (a) high HSV-2 challenge dose and (b) the 48-hour time point used for viral challenge in the study by Harandi et al. (6). Further, our finding that individual lymphocyte subsets and IFN- $\gamma$ R are dispensable for CPG ODN-mediated protection against HSV-2 is consistent with previous reports showing that RAG2-/- (lacking B and T cells), RAG2-/- $\gamma \mathrm{c}^{-/-}$(lacking B, T, NK, and NKT cells) (9), and IFN $\gamma^{-/-}$(5) mice were all protected from HSV-2 challenge following $\mathrm{CPG}$ ODN inoculation.

One potential complication of interpreting data from the irradiation-induced BM chimera stems from the radioresistant nature of the Langerhans cells (19). If Langerhans cells in the vaginal epithelia were repopulated from radioresistant host precursors, our interpretation must consider the contributions of Langerhans cells reflecting the host genotype. However, unlike the Langerhans cells that reside in the skin epidermis, vaginal Langerhans cells are repopulated entirely from BM precursors upon reconstitution of the irradiation-induced BM chimera (N. Iijima, M. Linehan, and A. Iwasaki, unpublished observations). Thus, in the WT $\rightarrow \mathrm{TLR}^{-/-}$mice, the vaginal Langerhans cells are of WT origin (TLR9'), and the reduction in the viral titers in these mice compared with the TLR9-/- mice reflects the contribution of the hematopoietic cells, including the vaginal Langerhans cells in their response to CPG ODN. In a similar vein, the observation that IFN $\alpha \beta \mathrm{R}^{-1-} \rightarrow$ WT mice succumbed to more severe disease compared with the WT $\rightarrow \mathrm{IFN} \alpha \beta \mathrm{R}^{-/-}$mice following CPG ODN treatment indicates the importance of the hematopoietic cells, including vaginal Langerhans cells, in responding to type I IFNs and providing antiviral protection in situ.

Our data demonstrate the importance of the responsiveness to type I IFNs by both the stromal and hematopoietic cells in providing $\mathrm{CpG}$ ODN-mediated antiviral protection in the genital mucosa. The requirement for IFN- $\alpha \beta$ R on the vaginal epithelial cells, the target of HSV-2 (16), was not surprising since signals transduced by the engagement of this receptor via type I IFNs result in the expression of hundreds of antiviral genes necessary to inhibit viral replication and spreading $(14,15)$. It is interesting to note that the level of viral suppression in the vaginal mucosa achieved in $\mathrm{CpG}$ $\mathrm{ODN}$-treated $\mathrm{WT} \rightarrow \mathrm{IFN} \alpha \beta \mathrm{R}^{-/-}$chimeric mice was comparable to that of CPG ODN-treated WT mice (Figure 6C). These data indicate that pDCs might provide type I IFN-independent antiviral activities for vaginal keratinocytes. However, the mechanism still remains unclear. It may involve other antiviral cytokines, NKmediated lysis of infected cells, or direct lysis of the infected cells by $\mathrm{pDCs}$, as shown by a recent study demonstrating that $\mathrm{pDCs}$
A

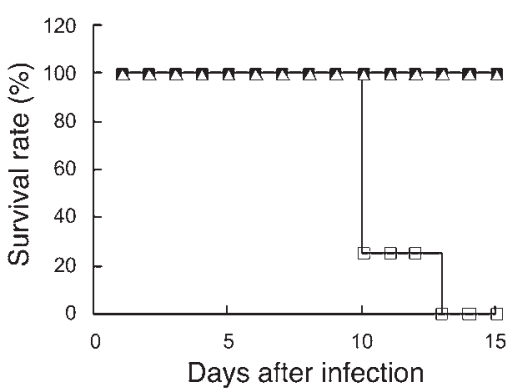

B

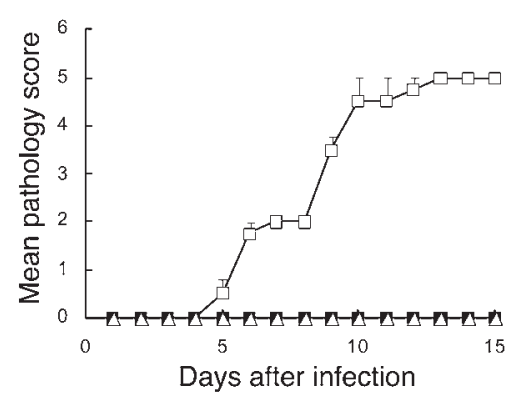

C

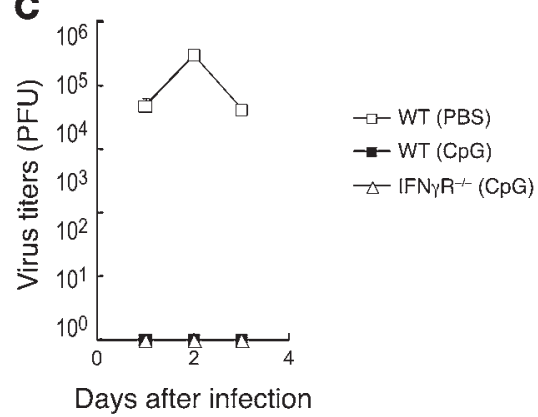

Figure 8

IFN- $\gamma$ responsiveness is not required for CpG ODN-mediated protection against genital HSV-2 challenge. (A) Survival rates, (B) genital mean pathology scores, and (C) virus titers in vaginal washes of WT (129 background) and IFN $\gamma \mathrm{R}^{-/-}$(129 background) mice were monitored following CpG ODN ivag inoculation and HSV-2 challenge (104 PFU). Values represent mean \pm SEM $(n=5 /$ group). Data are representative of 3 independent experiments. 
acquire cytolytic activities through expression of TNF-related apoptosis-inducing ligand upon CPG ODN stimulation (20).

The basis for the requirement for IFN responsiveness by the hematopoietic cells in providing this innate protection was less obvious. Among other possible explanations, it is important to note that IFN $\alpha \beta \mathrm{R}^{-/-}$pDCs fail to secrete robust type I IFNs (ref. 21 and data not shown). Like many other cell types, pDCs require positive feedback through the IFN- $\alpha \beta R$ (22) for production of high levels of type I IFNs, owing to the requirement for the IFNinduced IFN regulatory factor 7 (IRF-7) in this process (21). Thus, in the IFN $\alpha \beta \mathrm{R}^{-/-} \rightarrow$ WT chimera, one of the major defects in CPG ODN-mediated antiviral protection is the lack of $\mathrm{pDC}$ secretion of type I IFNs. In addition, type I IFNs are a potent activator of NK cytotoxicity (23). Combined with our data indicating that NK depletion had a moderate defect in viral control by CPG ODN treatment (Figure 3), the lack of protection seen in the IFN $\alpha \beta \mathrm{R}^{-/-} \rightarrow$ WT chimera may also reflect reduced NK cell activities.

Collectively, our results contribute to the understanding of several key mechanisms of $\mathrm{CPG}$ ODN-based treatment in providing an innate antiviral immune state. These findings suggest that $\mathrm{CpG}$ ODN-based microbicidal approaches may benefit from proper targeting of $\mathrm{CpG}$ ODN to the pDCs and to the vaginal epithelial cells. Such selective targeting of CPG ODN is expected to reduce the amount of $\mathrm{CPG}$ ODN required for effective microbicidal activity and also diminish inflammation that is associated with general application of TLR agonists (24) without compromising the effectiveness of CPG ODN as a microbicide. Finally, this study reveals the importance of the intricate crosstalk between $\mathrm{pDCs}$ and vaginal stromal cells in orchestrating innate antiviral protection upon local delivery of CPG ODN through type I IFNs.

\section{Methods}

Virus and CPG ODN. HSV-2 186 syn (25) was propagated and titrated as described previously (16). CPG 1826 ODN (5'-TCCATGACGTTCCTGACGTT-3') with phosphorothioate backbone (26) was synthesized and HPLCpurified by TriLink BioTechnologies.

Mice and virus infection. Six to eight-week-old female C57BL/6 and 129 mice were purchased from the National Cancer Institute, and IL-12p $40^{-/-}$mice on C57BL/6 background were purchased from Jackson Laboratory. MyD88-/mice (27) and TLR9 $/-$ mice (28) backcrossed onto a C57BL/6 background and IFN $\alpha \beta \mathrm{R}^{-/-}$mice (29) and IFN $\gamma \mathrm{R}^{-/-}$mice (30) backcrossed onto a 129 background were used. For virus infection, mice were injected s.c. in the neck ruff with Depo-Provera (Pfizer) at $2 \mathrm{mg}$ per mouse in a $100 \mu \mathrm{l}$ volume 5 days before infection, swabbed with calcium alginate, and inoculated ivag with $10^{4} \mathrm{PFU}$ of HSV-2 strain 186 syn in a $10 \mu \mathrm{l}$ volume by a blunt-ended micropipette tip. For CPG ODN treatment, mice were inoculated with $100 \mu \mathrm{g}$ of CpG ODN in $10 \mu \mathrm{l}$ volume ivag 24 hours before viral infection. This procedure was carried out according to a previous report demonstrating that the optimal period of protection was from -24 hours to 4 hours after CPG ODN treatment $(6,31)$. The severity of disease was scored (32) as follows: 0 , no sign; 1 , slight genital erythema and edema; 2 , moderate genital inflammation; 3 , purulent genital lesions; 4 , hind limb paralysis; 5 , premoribund. Due to humane concerns, the animals were euthanized prior to reaching moribund state. All procedures used in this study complied with federal guidelines and were approved by the Yale University Institutional Animal Care and Use Committee.

Construction of BM chimeras. BM chimeric mice were constructed by a standard method described previously (3). In brief, recipient mice were lethally irradiated with 9 Gy of total-body irradiation by Cs-irradiator (Yale Cancer Center). BM was obtained from the femur and tibia of donor mice and collected in sterile PBS. The cells were subsequently washed twice, counted, and resuspended in sterile PBS at a concentration of $5.0 \times 10^{7}$ cells $/ \mathrm{ml}$. Irradiated recipient mice were reconstituted with $1.0-1.5 \times 10^{7}$ cells by means of tail vein injection. The transplanted mice were treated with antibiotics and maintained in a clean facility and used for experiments upon complete reconstitution (>8 weeks). BM reconstitution was confirmed as previously described (3).

Collection of vaginal secretions and virus titration. Vaginal secretions were collected from -1 to 5 days after infection by pipetting a total volume of $50 \mu \mathrm{l}$ of PBS in and out of the vagina 20 times. Then the vaginal surface was swabbed twice. The vaginal swabs were left in the vaginal washes. Virus titers in the vaginal washes were determined by infecting confluent Vero cells for 2 to 3 days in triplicate by a standard method (16).

$p D C, N K$ cell, and T lymphocyte depletion. pDCs were depleted at 24 hours before and 24 hours after the $\mathrm{CPG}$ ODN treatment by i.p. injection of $500 \mu \mathrm{g}$ anti-mPDCA-1 in a volume of $500 \mu \mathrm{l} \mathrm{PBS} \mathrm{(rat} \mathrm{IgG2b;} \mathrm{Miltenyi} \mathrm{Biotec)}$ according to the manufacturer's instructions. NK cells were depleted 3 times at $-3,-1$, and 1 day after infection by i.p. injection of $200 \mu \mathrm{g}$ in $200 \mu \mathrm{l}$ of anti-NK1.1 (Mouse IgG2a). CD4 and CD8 T cells were depleted at -3, -1 , and 1,3 , and 5 days after infection by i.p injection of $400 \mu \mathrm{g}$ anti-CD4 (GK1.5; rat IgG2b) and anti-CD8 (53-6.72; rat IgG2a) Abs. Mice injected with rat IgG $(500 \mu \mathrm{g})$ or mouse IgG $(200 \mu \mathrm{g})$ at $-3,-1$, and 1 day after infection were used as controls. All depletion procedures followed a standard protocol, and in vivo depletion was confirmed by FACS analysis of the splenocytes from the Ab-treated mice.

Isolation of cells from vaginal tissues and FACS analysis. Vaginal tissues from mice treated with PBS or CPG ODN were excised and washed with PBS once. Tissues were incubated with $1 \mathrm{ml}$ of $2.0 \mathrm{U} / \mathrm{ml}$ Dispase II (Roche Diagnostics) PBS solution at $37^{\circ} \mathrm{C}$ for 1 hour. After removal of Dispase II solution, tissues were minced and incubated with $5 \mathrm{ml}$ of $0.05 \%$ Trypsin-EDTA PBS solution at $37^{\circ} \mathrm{C}$ for 20 minutes. After incubation, tissues were pressed through a $70-\mu \mathrm{m}$ cell strainer. Cells were collected and washed 3 times with $5 \mathrm{ml} /$ vagina of PBS solution containing $0.05 \%$ FBS. Cells were stained with PE-conjugated anti-mPDCA-1 Ab (or isotype control) and 7-AAD (BD Biosciences), and live cells were gated and analyzed by FACS.

Statistics. Statistical differences of the viral titers were determined by the ANOVA method. A $P$ value of less than 0.05 was considered statistically significant.

\section{Acknowledgments}

The authors are grateful for assistance provided by Heungkyu Lee, Melodie Zamora, Ayuko Sato, Jennifer Lund, Norifumi Iijima, and Melissa Linehan; helpful suggestions and support by Mark W. Saltzman; and critical review of the manuscript by Ruslan Medzhitov. This work was supported by Public Health Service grants AI054359, AI064705 (to A. Iwasaki), and EB000487 (to H. Shen via Mark Saltzman) from the NIH. This study was also partly supported by Yale University's Center for Interdisciplinary Research on AIDS through a grant from the National Institute of Mental Health (P30 MH 62294 to Michael Merson).

Received for publication March 29, 2006, and accepted in revised form June 6, 2006.

Address correspondence to: Akiko Iwasaki, Section of Immunobiology, Yale University School of Medicine, 300 Cedar Street, New Haven, Connecticut 06520, USA. Phone: (203) 785-2919; Fax: (203) 737-1764; E-mail: akiko.iwasaki@yale.edu.

Hong Shen's present address is: Department of Chemical Engineering, University of Washington, Seattle, Washington, USA. 
1. Stone, A. 2002. Microbicides: a new approach to preventing HIV and other sexually transmitted infections. Nat. Rev. Drug Discov. 1:977-985.

2. Iwasaki, A., and Medzhitov, R. 2004. Toll-like receptor control of the adaptive immune responses. Nat. Immunol. 5:987-995.

3. Sato, A., and Iwasaki, A. 2004. Induction of antiviral immunity requires Toll-like receptor signaling in both stromal and dendritic cell compartments. Proc. Natl. Acad. Sci. U. S. A. 101:16274-16279.

4. Ashkar, A.A., et al. 2004. Toll-like receptor (TLR)-3, but not TLR4, agonist protects against genital herpes infection in the absence of inflammation seen with CpG DNA. J. Infect. Dis. 190:1841-1849.

5. Ashkar, A.A., Bauer, S., Mitchell, W.J., Vieira, J., and Rosenthal, K.L. 2003. Local delivery of CpG oligodeoxynucleotides induces rapid changes in the genital mucosa and inhibits replication, but not entry, of herpes simplex virus type 2. J. Virol. 77:8948-8956.

6. Harandi, A.M., Eriksson, K., and Holmgren, J. 2003. A protective role of locally administered immunostimulatory $\mathrm{CPG}$ oligodeoxynucleotide in a mouse model of genital herpes infection. J. Virol. 77:953-962.

7. Pyles, R.B., et al. 2002. Use of immunostimulatory sequence-containing oligonucleotides as topical therapy for genital herpes simplex virus type 2 infection. J. Virol. 76:11387-11396.

8. Sajic, D., Patrick, A.J., and Rosenthal, K.L. 2005. Mucosal delivery of $\mathrm{CpG}$ oligodeoxynucleotides expands functional dendritic cells and macrophages in the vagina. Immunology. 114:213-224.

9. Gill, N., Rosenthal, K.L., and Ashkar, A.A. 2005. NK and NKT cell-independent contribution of interleukin-15 to innate protection against mucosal viral infection. J. Virol. 79:4470-4478.

10. Parr, M.B., et al. 1994. A mouse model for studies of mucosal immunity to vaginal infection by herpes simplex virus type 2. Lab. Invest. 70:369-380.

11. Medzhitov, R., et al. 1998. MyD88 is an adaptor protein in the hToll/IL-1 receptor family signaling pathways. Mol. Cell. 2:253-258.

12. Colonna, M., Trinchieri, G., and Liu, Y.J. 2004. Plasmacytoid dendritic cells in immunity. Nat. Immunol. 5:1219-1226.

13. Liu, Y.J. 2005. IPC: professional type 1 interferonproducing cells and plasmacytoid dendritic cell precursors. Annu. Rev. Immunol. 23:275-306.

14. Katze, M.G., He, Y., and Gale, M., Jr. 2002. Viruses and interferon: a fight for supremacy. Nat. Rev Immunol. 2:675-687.

15. Taniguchi, T., and Takaoka, A. 2002. The interferon-alpha/beta system in antiviral responses: a multimodal machinery of gene regulation by the IRF family of transcription factors. Curr. Opin. Immunol. 14:111-116.

16. Zhao, X., et al. 2003. Vaginal submucosal dendritic cells, but not Langerhans cells, induce protective Th1 responses to herpes simplex virus-2. J. Exp. Med. 197:153-162.

17. Akira, S. 2001. Toll-like receptors and innate immunity. Adv. Immunol. 78:1-56.

18. Medzhitov, R. 2001. Toll-like receptors and innate immunity. Nat. Rev. Immunol. 1:135-145.

19. Merad, M., et al. 2002. Langerhans cells renew in the skin throughout life under steady-state conditions. Nat. Immunol. 3:1135-1141.

20. Chaperot, L., et al. 2006. Virus or TLR agonists induce TRAIL-mediated cytotoxic activity of plasmacytoid dendritic cells. J. Immunol. 176:248-255.

21. Honda, K., et al. 2005. IRF-7 is the master regulator of type-I interferon-dependent immune responses. Nature. 434:772-777.
22. Sato, M., et al. 1998. Positive feedback regulation of type I IFN genes by the IFN-inducible transcription factor IRF-7. FEBS Lett. 441:106-110.

23. Biron, C.A., Nguyen, K.B., Pien, G.C., Cousens, L.P., and Salazar-Mather, T.P. 1999. Natural killer cells in antiviral defense: function and regulation by innate cytokines. Annu. Rev. Immunol. 17:189-220.

24. Bernstein, D.I., Spruance, S.L., Arora, S.S., Schroeder, J.L., and Meng, T.C. 2005. Evaluation of imiquimod $5 \%$ cream to modify the natural history of herpes labialis: a pilot study. Clin. Infect. Dis. 41:808-814.

25. Gao, M., Bouchey, J., Curtin, K., and Knipe, D.M. 1988. Genetic identification of a portion of the herpes simplex virus ICP8 protein required for DNAbinding. Virology. 163:319-329.

26. Krieg, A.M., et al. 1995. CpG motifs in bacterial DNA trigger direct B-cell activation. Nature. 374:546-549.

27. Adachi, O., et al. 1998. Targeted disruption of the MyD88 gene results in loss of IL-1- and IL-18-mediated function. Immunity. 9:143-150.

28. Hemmi, H., et al. 2000. A Toll-like receptor recognizes bacterial DNA. Nature. 408:740-745.

29. Muller, U., et al. 1994. Functional role of type I and type II interferons in antiviral defense. Science. 264:1918-1921.

30. Huang, S., et al. 1993. Immune response in mice that lack the interferon-gamma receptor. Science. 259:1742-1745.

31. Sajic, D., et al. 2003. Parameters of CpG oligodeoxynucleotide-induced protection against intravaginal HSV-2 challenge. J. Med. Virol. 71:561-568.

32. Morrison, L.A., Da Costa, X.J., and Knipe, D.M. 1998. Influence of mucosal and parenteral immunization with a replication-defective mutant of HSV-2 on immune responses and protection from genital challenge. Virology. 243:178-187. 